Pacific Journal of Mathematics

ON THE UNIFORM DISTRIBUTION PROPERTY OF CERTAIN 


\title{
ON THE UNIFORM DISTRIBUTION PROPERTY OF CERTAIN LINEAR ALGEBRAIC GROUPS
}

\author{
Atsushi Murase
}

\begin{abstract}
Let $G$ be a connected semisimple linear algebraic group defined over an algebraic number field $k$. Denote by $G_{k}$ and $G_{A}$ the group of $k$-rational points of $G$ and its adelization. In this paper, we prove, under suitable assumptions on $G$, a uniformity of distribution of $G_{k}$ in $G_{A}$ with respect to the Haar measure on $G_{A}$.
\end{abstract}

Introduction. Let $G$ be a connected semisimple linear algebraic group defined over an algebraic number field $k$. We denote by $G_{k}$ the group of $k$-rational points of $G$, and we write $G_{A}$ for its adelization.

The purpose of this paper is to show, under suitable assumptions on $G$, that $G_{k}$ is, in a sense, "uniformly distributed" in $G_{A}$ with respect to a Haar measure on $G_{A}$.

For each place $v$ of $k$, let $G_{k_{v}}$ be the group of $k_{v}$-rational points of $G$ where $k_{v}$ is the $v$-completion of $k$. If $v$ is a finite place, let $O_{v}$ be the maximal compact subring of $k_{v}$. Then $G_{o_{v}}$, the group of $O_{v}$-rational points of $G$, is an open compact subgroup of $G_{k_{v}}$. We set

$$
\begin{gathered}
G_{\infty}=\prod_{v \in \mathscr{S}_{\infty}} G_{k_{v}}, \\
G_{\boldsymbol{A}_{f}}=\prod_{v \in \mathscr{S}_{f}}^{\prime} G_{k_{v}} \text { (restricted direct product). }
\end{gathered}
$$

Here $\mathscr{P}_{\infty}\left(\right.$ resp. $\left.\mathscr{P}_{f}\right)$ denotes the set of all infinite (resp. finite) places of $k$. Then we have

$$
G_{A}=G_{A_{f}} G_{\infty} \text { (direct product). }
$$

Let $\mathscr{S}$ be a finite subset of $\mathscr{P}_{f}$. Furthermore, for each $\mathfrak{g} \in \mathscr{S}$, let $K_{8}$ be an open compact subgroup of $G_{o_{g}}$ and let $\left\{S_{8}(j)\right\}_{j=1}^{\infty}$ be a sequence of nonempty compact subsets of $G_{k_{8}}$ satisfying the following conditions:

$$
K_{8} S_{8}(j) K_{8}=S_{8}(j) \quad(j=1,2, \cdots) .
$$

We set

$$
S(j)=\prod_{g \in \mathscr{S}} S_{8}(j) \times \prod_{g \in \mathscr{S}_{f}^{-S}} G_{o_{g}} .
$$

Then $S(j)$ is a compact subset of $G_{A_{f}}$. For a relatively compact 
domain $S_{\infty}$ in $G_{\infty}$, let $N\left(S(j), S_{\infty}\right)$ be the number of points in the set $\left(S(j) \times S_{\infty}\right) \cap G_{k}$. It is easy to see that $N\left(S(j), S_{\infty}\right)$ is finite.

We say that a sequence $\{S(j)\}_{j=1}^{\infty}$ has the uniform distribution property with respect to a Haar measure $d g$ on $G_{A}$ if the following equality holds for any relatively compact domain $S_{\infty}$ in $G_{\infty}$ :

$$
\lim _{j \rightarrow \infty} N\left(S(j), S_{\infty}\right) / \int_{S(j) \times S_{\infty}} d g=1 / \int_{G k \mid G_{A}} d g \text {. }
$$

Note that the above statement does not depend on the choice of a Haar measure on $G_{A}$.

Let $d g_{f}$ be a Haar measure on $G_{A_{f}}$. Then our main result is stated as follows.

THEOREM 1. Notation being as above, assume that $G$ is anisotropic (namely that $G_{k} \mid G_{A}$ is compact). Furthermore, we assume that $G$ is absolutely almost simple $e^{1)}$ and simply connected $d^{2)}$. Then the sequence $\{S(j)\}_{j=1}^{\infty}$ has the uniform distribution property with respect to a Haar measure $d g$, if the equality (0.1) is satisfied:

$$
\lim _{j \rightarrow \infty} \int_{S(j)} d g_{f}=+\infty \text {. }
$$

REMARK 1. The additional assumption that $G$ is absolutely almost simple can be replaced by the following weaker assumption (A).

(A) For $g \in \mathscr{S}$, if $G_{k_{\mathrm{g}}}$ is noncompact then $G$ is $k_{\mathrm{g}}$-almost simple (namely that $G$ has no proper closed connected normal subgroups defined over $k_{9}$ ) and $G_{k} G_{k_{8}}$ is dense in $G_{A}$.

Note that $G$ has the property (A) if $G$ is absolutely almost simple and simply connected, by virtue of the strong approximation theorem (cf. [9], [11], and [12]).

REMARK 2. There are numbers of examples of $G$ satisfying the assumptions in Theorem 1 (e.g., quaternion unitary groups constructed by G. Shimura in [14]).

Even if $G$ is not anisotropic, it is probable that an analogue of Theorem 1 is available. At present we can prove only the following:

THeOREM 2. Let $G$ be $\mathrm{SL}_{2}$ (regarded as a linear algebraic group defined over $k$ ). Then the sequence $\{S(j)\}_{j=1}^{\infty}$ has the uniform distribution property if (0.1) is satisfied.

We present an implication of our results. Assume that $G$

1) This implies that $G$ has no proper closed connected normal subgroups.

2) For the definition, see [8], p. 189. 
satisfies the assumptions in Theorem 1 . Assume $k=\boldsymbol{Q}$ and $\mathscr{S}=$ $\{p\}^{3)}$. Let $S_{j}$ be the set of elements $g$ in $G_{Q}$ such that the coordinates of $p^{j} g$ are integral and moreover satisfy some prescribed congruence conditions. Let $U, V$ be relatively compact domains in $G_{R}$, and let $v(U), v(V)$ be their volumes measured by a Haar measure on $G_{\boldsymbol{R}}$. We denote by $N_{U}(j)$ (resp. $N_{V}(j)$ ) the number of elements in the set $U \cap S_{j}$ (resp. $V \cap S_{j}$ ). Then we have

$$
\lim _{j \rightarrow \infty} N_{U}(j) / N_{V}(j)=v(U) / v(V)^{4)} .
$$

A special case of Theorem 1 was first obtained by M. Kuga in [10] when $G$ is the group of indefinite division quaternions with reduced norm $1^{5)}$. Several ideas in [10] together with recent results of Howe and Moore [7] are basic in our present paper.

Note that H. Yoshida shows in [16] that Theorem 2 for $k=\boldsymbol{Q}$ leads to his distribution law for PSL $(2, Z[1 / p])$-elliptic conjugacy classes.

I would like to express here my deep gratitude to Professor T. Shintani for his suggestion of the problem considered here, his many mathematical and linguistical advices and his constant encouragement during the preparation of this paper.

Notation. For a complex number $s$, we denote by $\operatorname{Im} s$ (resp. Re $s$ ) the imaginary (resp. real) part of $s$. For an algebraic number field $k$, we denote by $\boldsymbol{A}$ and $\boldsymbol{I}$ the adele ring of $k$ and the idele group of $k$, respectively. We denote by $|a|_{A}$ the module of an idele $a$, given by the equality $d(a x)=|a|_{A} d x$ where $d x$ is a Haar measure on $\boldsymbol{A}$. For a locally compact topological space $X$, we denote by $C^{0}(X)$ the space of continuous functions on $X$ and denote by $C_{c}^{0}(X)$ the space consisting of $f \in C^{0}(X)$ with compact support. For

3) We assume that $G_{Q_{p}}$ is noncompact.

4) In fact, our result implies the following asymptotic formula for $N_{U}(j)$. Let $K$ be a sufficiently small open compact subgroup of $\Pi_{l<\infty} G_{i} Z_{l}$, and put $I^{\prime}=\left(K \times G_{R}\right) \cap$ GQ. We may assume that $\Gamma S_{j} \Gamma=S_{j}(j=1,2, \cdots)$. We denote by $\left|\Gamma \backslash S_{j}\right|$ the number of left $\Gamma$-cosets contained in $S_{j}$. Then we have

$$
N_{U}(j) \sim \operatorname{vol}\left(\Gamma \backslash G_{R}\right)^{-1} \cdot v(U) \cdot\left|\Gamma \backslash S_{j}\right|
$$

as $j \rightarrow \infty$.

5) C. Pommerenke obtained in [13] the following similar results, while his method seems to be different from ours.

Let $A$ be a positive definite symmetric integral matrix of size $m \geqq 5$. Set $X=$ $\left\{x \subseteq \boldsymbol{R}^{m} \mid{ }^{t} x A x=1\right\} \subset \boldsymbol{R}^{m}$. For a positive integer $n$, put

$$
S_{n}=\left\{\xi / \sqrt{n} \mid \xi \in Z^{m},{ }^{t} \xi A \xi=n\right\} \subset X .
$$

Let $A$ be the set consisting of positive integers $n$ such that $S_{n} \div \dot{\tau}$. Then the sequence $\left\{S_{n}\right\}_{n \in A}$ is uniformly distributed in $X$ with respect to a suitable measure on $X$. 
a continuous function $f$ on a locally compact group $G$, and for a compact subgroup $M$ of $G$, we say that $f$ is right $M$-finite if the set $\left\{R_{m} f ; m \in M\right\}$ spans a finite dimensional subspace in $C^{0}(G)$, where we set $R_{m} f(g)=f(g m)$. For a bounded linear operator $T$ on a Hilbert space $H$, we denote by $\|T\|$ the operator norm of $T$, given by

$$
\|T\|=\sup _{v \in H, v \neq 0}\|T v\| /\|v\| .
$$

For a finite dimensional vector space $V$ over $C$, we denote by $\operatorname{End}_{c}(V)$ the $\boldsymbol{C}$-algebra of $\boldsymbol{C}$-endomorphisms on $V$. If $T$ is a $\boldsymbol{C}$ endomorphism on a $\boldsymbol{C}$-vector space with an inner product, we denote by $T^{*}$ the adjoint of $T$ with respect to the inner product. If $\tau$ is an unitary representation of a compact group $M$ on a finite dimensional vector space $V$ over $C$, we set $\operatorname{dim} \tau=\operatorname{dim}_{c} V$.

1. We keep notation in the introduction without further comment. From now on, we always assume that $G$ is a connected semisimple linear algebraic group defined over an algebraic number field $k$. We set

$$
K=\prod_{\mathfrak{g} \in \mathscr{S}_{f}^{-S}} G_{O_{\mathfrak{g}}} \times \prod_{\mathfrak{g} \in \mathscr{S}} K_{\mathfrak{g}} .
$$

Then $K$ is an open compact subgroup of $G_{A_{f}}$. We normalize the Haar measure $d g_{f}$ on $G_{A_{f}}$ so that

$$
\int_{K} d g_{f}=1
$$

Choose a Haar measure $d g_{\infty}$ on $G_{\infty}$ and fix the Haar measure $d g$ on $G_{A}$ by setting

$$
d g=d g_{f} d g_{\infty}\left(g=g_{f} g_{\infty}, g_{f} \in G_{A_{f}}, g_{\infty} \in G_{\infty}\right) .
$$

Then $d g$ induces an invariant measure $d \dot{g}$ on $G_{k} \mid G_{A}$ in a natural manner.

Let $L^{2}\left(G_{k} \mid G_{A} / K\right)$ be the Hilbert space of right $K$-invariant square integrable functions on $G_{k} \mid G_{A}$. Note that constant functions are in $L^{2}\left(G_{k} \mid G_{A} / K\right)$, since the volume of the quotient space $G_{k} \mid G_{A}$ is finite (cf. [2], 5.6).

Let $\xi_{j}$ be the characteristic function of $S(j)$. Then $\xi_{j}$ is $K$ biinvariant, continuous, and compactly supported on $G_{A_{f}}$. For each $f \in L^{2}\left(G_{k} \mid G_{A} / K\right)$, set

$$
f * \xi_{j}(g)=\int_{G_{A_{f}}} f\left(g h_{f}^{-1}\right) \xi_{j}\left(h_{f}\right) d h_{f} .
$$

Then the mapping $f \mapsto f * \xi_{j}$ gives rise to a bounded linear operator 
on $L^{2}\left(G_{k} \mid G_{A} / K\right)$. We set

$$
\operatorname{deg} \xi_{j}=\int_{G_{A_{f}}} \xi_{j}\left(g_{f}\right) d g_{f}=\int_{S(j)} d g_{f} .
$$

Then $\operatorname{deg} \xi_{j}$ is equal to the 'number of left $K$-cosets contained in $S(j)$. We denote by \|\| and $($, ) the norm and the inner product in $L^{2}\left(G_{k} \mid G_{A} / K\right)$, respectively. Set

$$
v=\int_{G_{k} \backslash G_{A}} d \dot{g} .
$$

Then the following proposition plays a basic role in the present paper.

Proposition 1. The sequence $\{S(j)\}_{j=1}^{\infty}$ has the uniform distribution property with respect to a Haar measure on $G_{A}$, if the following equality holds for any $f \in L^{2}\left(G_{k} \mid G_{A} / K\right)$;

$$
\lim _{j \rightarrow \infty}\left\|f * \xi_{j} / \operatorname{deg} \xi_{j}-(f, 1) / v\right\|=0 .
$$

To prove the proposition, we need the next lemma.

Lemma 1. Under the assumption of Proposition 1, we have, for any $\left.\varphi \in C_{c}^{0}\left(G_{k}\right) G_{A} / K\right)$ and $g \in G_{A}$,

$$
\lim _{j \rightarrow \infty} \varphi * \xi_{j}(g) / \operatorname{deg} \xi_{j}=(\varphi, 1) / v .
$$

Proof. Assume that the lemma is false. Then there exists $g_{0} \in G_{A}$ such that the equality (1.4) does not hold for $g_{0}$. We have

$$
\limsup _{j \rightarrow \infty}\left|\varphi * \xi_{j}\left(g_{0}\right) / \operatorname{deg} \xi_{j}-(\varphi, 1) / v\right|=\eta>0 .
$$

We can choose a subsequence $\left\{\xi_{j_{k}}\right\}_{k=1}^{\infty}$ of $\left\{\xi_{j}\right\}_{j=1}^{\infty}$ such that

$$
\lim _{k \rightarrow \infty}\left|\varphi * \xi_{j_{k}}\left(g_{0}\right) / \operatorname{deg} \xi_{j_{k}}-(\varphi, 1) / v\right|=\eta .
$$

Let $S(j)=\sum_{l=1}^{N_{j}} K \sigma_{l}^{(j)}\left(\sigma_{l}^{(j)} \in G_{A_{f}}\left(1 \leqq l \leqq N_{j}\right)\right)$ be a decomposition of $S(j)$ into a disjoint union of left $K$-cosets. (The number $N_{j}$ equals $\operatorname{deg} \xi_{j}$.) It is easily verified that

$$
\varphi * \xi_{j}(g)=\sum_{i=1}^{N_{j}} \varphi\left(g \sigma_{l}^{(j)-1}\right) .
$$

Hence we have, for $g \in G_{A}$,

$$
\begin{aligned}
& \left|\varphi * \xi_{j_{k}}(g) / \operatorname{deg} \xi_{j_{k}}-(\varphi, 1) / v\right| \\
& \quad \geqq\left|\varphi * \xi_{j_{k}}\left(g_{0}\right) / \operatorname{deg} \xi_{j_{k}}-(\varphi, 1) / v\right|
\end{aligned}
$$




$$
\begin{aligned}
& -\frac{1}{N_{j_{k}}}\left|\varphi * \xi_{j_{k}}(g)-\varphi * \xi_{j_{k}}\left(g_{0}\right)\right| \\
& \geqq\left|\varphi * \xi_{j_{k}}\left(g_{0}\right) / \mathrm{deg} \xi_{j_{k}}-(\varphi, 1) / v\right| \\
& -\frac{1}{N_{j_{k}}} \sum_{l=1}^{N_{j_{k}}}\left|\varphi\left(g \sigma_{l}^{(j)-1}\right)-\varphi\left(g_{0} \sigma_{l}^{(j)-1}\right)\right| .
\end{aligned}
$$

Since the function $\varphi$ is continuous and compactly supported on $G_{k} \mid G_{A}$, there exists an open neighborhood $U$ of 1 in $G_{A}$ such that $g_{1}^{-1} g_{2} \in U$ always implies

$$
\left|\varphi\left(g_{1}\right)-\varphi\left(g_{2}\right)\right|<\eta / 2 .
$$

Suppose that $g \in g_{0}\left(G_{\infty} \cap U\right)$. Then we have $\left(g_{0} \sigma_{l}^{\left(j_{k}\right)-1}\right)^{-1} \times\left(g \sigma_{l}^{\left(j_{k}\right)-1}\right)=$ $\sigma_{l}^{\left(j_{k}\right)} g_{0}^{-1} g \sigma_{l}^{\left(j_{k}\right)-1}=g_{0}^{-1} g \in U$. (Note that $g_{0}^{-1} g \in G_{\infty}$ commutes with every element in $G_{A_{f}}$. $)$ Thus we have, for $g \in g_{0}\left(G_{\infty} \cap U\right)$,

$$
\frac{1}{N_{j_{k}}} \sum_{l=1}^{N_{j_{k}}}\left|\varphi\left(g \sigma_{l}^{\left(j_{j_{k}}\right)-1}\right)-\varphi\left(g_{0} \sigma_{l}^{\left(j_{k}\right)-1}\right)\right|<\eta / 2 .
$$

Hence, for any $g \in g_{0}\left(G_{\infty} \cap U\right)$, the following inequality holds:

$$
\begin{gathered}
\liminf _{k \rightarrow \infty}\left|\varphi * \xi_{j_{k}}(g) / \operatorname{deg} \xi_{j_{k}}-(\varphi, 1) / v\right| \\
\geqq \eta-\eta / 2=\eta / 2 .
\end{gathered}
$$

In fact, this inequality (1.5) holds for any $g \in G_{k} g_{0}\left(G_{\infty} \cap U\right) K$ since $\varphi * \xi_{j_{k}}$ is left $G_{k}$-invariant and right $K$-invariant. Since $\left(G_{\infty} \cap U\right) K$ is an open set in $G_{A}$, we have, by virtue of Fatou's lemma,

$$
\begin{aligned}
& \liminf _{k \rightarrow \infty}\left\|\varphi * \xi_{j_{k}} / \operatorname{deg} \xi_{j_{k}}-(\varphi, 1) / v\right\|^{2} \\
& \quad \geqq \int_{G_{k} \backslash \epsilon_{k} g_{0}\left(G_{\infty} \cap U\right) K} \liminf _{k \rightarrow \infty}\left|\varphi * \xi_{j_{k}}(g) / \operatorname{deg} \xi_{j_{k}}-(\varphi, 1) / v\right|^{2} d \dot{g} \\
& \quad \geqq(\eta / 2)^{2} \int_{G_{k} \backslash G_{k} g_{0}\left(G_{\infty} \cap U\right) K} d \dot{g}>0 .
\end{aligned}
$$

Contradiction! The lemma has been established.

Proof of Proposition 1. Let us consider any two relatively compact domains $S_{\infty}^{\prime}, S_{\infty}^{\prime \prime}$ in $G_{\infty}$ satisfying $\overline{S_{\infty}^{\prime}} \subset S_{\infty}^{\prime \prime}$ (we denote by $\overline{S_{\infty}^{\prime}}$ the closure of $S_{\infty}^{\prime}$ in $G_{\infty}$ ). We choose a real-valued continuous function $\psi_{\infty}$ on $G_{\infty}$ satisfying the following conditions (1.6) and (1.7):

$$
\begin{aligned}
& 0 \leqq \psi_{\infty}\left(g_{\infty}\right) \leqq 1 \text { for any } g_{\infty} \in G_{\infty}, \\
& \psi_{\infty}\left(g_{\infty}\right)=\left\{\begin{array}{l}
1 \text { if } g_{\infty} \in S_{\infty}^{\prime} \\
0 \text { if } g_{\infty} \notin S_{\infty}^{\prime \prime} .
\end{array}\right.
\end{aligned}
$$

We set $\psi(g)=\psi_{f}\left(g_{f}\right) \psi_{\infty}\left(g_{\infty}\right)$ for $g=g_{f} g_{\infty}\left(g_{f} \in G_{A_{f}}\right.$ and $\left.g_{\infty} \in G_{\infty}\right)$, where 
$\psi_{f}$ denotes the characteristic function of $K$. Then $\psi(g)$ is continuous and compactly supported on $G_{A}$. It is easy to see that the series:

$$
\varphi(g)=\sum_{\gamma \in G_{k}} \psi(\gamma g)
$$

converges absolutely and uniformly ion 'any compact subset of $G_{A}$, and that $\varphi(g) \in C_{c}^{0}\left(G_{k} \mid G_{A} / K\right)$. Applying Lemma 1 to $\varphi$, we obtain

$$
\lim _{j \rightarrow \infty} \varphi * \xi_{j}(1) / \operatorname{deg} \xi_{j}=(\varphi, 1) / v .
$$

We have

$$
\begin{aligned}
(\varphi, 1) & =\int_{G_{k \backslash G_{A}}} \varphi(g) d \dot{g}=\int_{G_{A}} \psi(g) d g \\
& =\int_{G_{A_{f}}} \psi_{f}\left(g_{f}\right) d g_{f} \cdot \int_{G_{\infty}} \psi_{\infty}\left(g_{\infty}\right) d g_{\infty} .
\end{aligned}
$$

In view of the conditions (1.6) and (1.7) imposed on $\psi_{\infty}$, we have

$$
\mu\left(S_{\infty}^{\prime}\right) \leqq(\varphi, 1) \leqq \mu\left(S_{\infty}^{\prime \prime}\right),
$$

where we set $\mu\left(S_{\infty}^{(i)}\right)=\int_{S_{\infty}^{(i)}} d g_{\infty}(i=1,2)$.

Next we have

$$
\begin{aligned}
\varphi * \xi_{j}(1) & =\int_{G_{A_{f}}} \varphi\left(g_{f}^{-1}\right) \xi_{j}\left(g_{f}\right) d g_{f}=\int_{G_{A_{f}}} \sum_{\gamma \in G_{k}} \psi\left(\gamma g_{f}^{-1}\right) \xi_{j}\left(g_{f}\right) d g_{f} \\
& =\sum_{\gamma \in G_{k}} \psi_{\infty}\left(\gamma_{\infty}\right) \int_{G_{A_{f}}} \psi_{f}\left(\gamma_{f} g_{f}^{-1}\right) \xi_{j}\left(g_{f}\right) d g_{f},
\end{aligned}
$$

where we write $\gamma=\gamma_{f} \gamma_{\infty}\left(\gamma_{f} \in G_{A_{f}}\right.$ and $\left.\gamma_{\infty} \in G_{\infty}\right)$. Since $\psi_{f}\left(\gamma_{f} g_{f}^{-1}\right) \xi_{j}\left(g_{f}\right)$ is, as a function of $g_{f}$, the characteristic function of $K \gamma_{f} \cap S(j)$, we have

$$
\int_{G_{A_{f}}} \psi_{f}\left(\gamma_{f} g_{f}^{-1}\right) \xi_{j}\left(g_{f}\right) d g_{f}= \begin{cases}1 & \text { if } \gamma_{f} \in S(j) \\ 0 & \text { otherwise }\end{cases}
$$

Hence

$$
\varphi * \xi_{j}(1)=\sum_{\gamma \in G_{k} \cap\left(S(j) \times G_{\infty}\right)} \psi_{\infty}\left(\gamma_{\infty}\right) .
$$

Then (1.6) and (1.7) imply that

$$
\sum_{\gamma \in G_{k} \cap\left(S(j) \times S_{\infty}^{\prime}\right)} 1 \leqq \varphi * \xi_{j}(1) \leqq \sum_{\gamma \in G_{k} \cap\left(S(j) \times S_{\infty}^{\prime \prime}\right)} 1 .
$$

Thus,

$$
N\left(S(j), S_{\infty}^{\prime}\right) \leqq \varphi * \xi_{j}(1) \leqq N\left(S(j), S_{\infty}^{\prime \prime}\right) .
$$

Combining two inequalities (1.9) and (1.10), we get 


$$
\begin{aligned}
& N\left(S(j), S_{\infty}^{\prime}\right) / \mu\left(S_{\infty}^{\prime \prime}\right) \operatorname{deg} \xi_{j} \leqq \varphi * \xi_{j}(1) /(\varphi, 1) \operatorname{deg} \xi_{j} \\
& \quad \leqq N\left(S(j), S_{\infty}^{\prime \prime}\right) / \mu\left(S_{\infty}^{\prime}\right) \operatorname{deg} \xi_{j} .
\end{aligned}
$$

It follows from (1.8) that

$$
\begin{array}{r}
\limsup _{j \rightarrow \infty} N\left(S(j), S_{\infty}^{\prime}\right) / \mu\left(S_{\infty}^{\prime \prime}\right) \operatorname{deg} \xi_{j} \leqq 1 / v \\
\leqq \\
\liminf _{j \rightarrow \infty} N\left(S(j), S_{\infty}^{\prime \prime}\right) / \mu\left(S_{\infty}^{\prime}\right) \operatorname{deg} \xi_{j} .
\end{array}
$$

It is easy to see

$$
\int_{S(j) \times S_{\infty}^{(i)}} d g=\mu\left(S_{\infty}^{(i)}\right) \cdot \operatorname{deg} \xi_{j} \quad(i=1,2) .
$$

Hence we get the following two inequalities;

$$
\begin{aligned}
& \limsup _{j \rightarrow \infty} N\left(S(j), S_{\infty}^{\prime}\right) / \int_{S(j) \times S_{\infty}^{\prime}} d g \leqq 1 / v \cdot \mu\left(S_{\infty}^{\prime \prime}\right) / \mu\left(S_{\infty}^{\prime}\right), \\
& \liminf _{j \rightarrow \infty} N\left(S(j), S_{\infty}^{\prime \prime}\right) / \int_{S(j) \times S_{\infty}^{\prime \prime}} d g \geqq 1 / v \cdot \mu\left(S_{\infty}^{\prime}\right) / \mu\left(S_{\infty}^{\prime \prime}\right) .
\end{aligned}
$$

For a given relatively compact domain $S_{\infty}$ in $G_{\infty}$, apply (1.11), setting $S_{\infty}^{\prime}=S_{\infty}$. For any $\varepsilon>0$, there exists a relatively compact domain $S_{\infty}^{\prime \prime}$ such that $1 \leqq \mu\left(S_{\infty}^{\prime \prime}\right) / \mu\left(S_{\infty}\right) \leqq 1+\varepsilon$ and that $S_{\infty}^{\prime \prime} \supset \overline{S_{\infty}}$. Hence we have

$$
\limsup _{j \rightarrow \infty} N\left(S(j), S_{\infty}\right) / \int_{S(j) \times S_{\infty}} d g \leqq 1 / v .
$$

Similar arguments for the inequality (1.12) (in this case, we set $S_{\infty}^{\prime \prime}=S_{\infty}$ ) lead to

$$
\liminf _{j \rightarrow \infty} N\left(S(j), S_{\infty}\right) / \int_{S(j) \times S_{\infty}} d g \geqq 1 / v .
$$

Thus we have

$$
\lim _{j \rightarrow \infty} N\left(S(j), S_{\infty}\right) / \int_{S(j) \times S_{\infty}} d g=1 / v .
$$

This implies that the sequence $\{S(j)\}_{j=1}^{\infty}$ has the uniform distribution property with respect to a Haar measure on $G_{A}$, and hence the proposition has been proved.

2. In this section, we assume that $G$ is anisotropic, simply connected, and satisfies the condition (A).

We shall prove the following:

Proposition 2. If (0.1) is satisfied, the equality 


$$
\lim _{j \rightarrow \infty}\left\|f * \xi_{j} / \operatorname{deg} \xi_{j}-(f, 1) / v\right\|=0
$$

holds for any $f \in L^{2}\left(G_{k} \mid G_{A} / K\right)$.

By virtue of Proposition 1, Theorem 1 is an immediate consequence of this result.

In this section we keep the normalization of Haar measures on $G_{A_{f}}, G_{\infty}$, and $G_{A}$ given in $\S 1$. We set $K_{g}=G_{O_{g}}$ for $\mathfrak{g} \in \mathscr{P}_{f}-\mathscr{S}$ and normalize the Haar measure $d g_{\mathfrak{g}}$ on $G_{k_{\mathfrak{g}}}$ for $\mathfrak{g} \in \mathscr{P}_{f}$ so that $\int_{K_{\mathfrak{g}}} d g_{\mathfrak{g}}=1$. The product measure $\Pi_{8 \in \mathscr{P}_{f}} d g_{8}$ is equal to the previously normalized Haar measure $d g_{f}$ on $G_{A_{f}}$.

Let $L^{2}\left(G_{k} \mid G_{A}\right)$ be the Hilbert space of square integrable functions on $G_{k} \mid G_{A}$. Then $L^{2}\left(G_{k} \mid G_{A} / K\right)$ is the closed subspace of right $K$-invariant functions in $L^{2}\left(G_{k} \mid G_{A}\right)$.

The next lemma is easily verified.

LEMMA 2. Let $\left\{T_{j}\right\}_{j=1}^{\infty}$ be a sequence of bounded linear operators on a Hilbert space $H$ such that

$$
\sup _{j \geqq 1}\left\|T_{j}\right\|<\infty .
$$

Let $\left\{F_{n}\right\}_{n=1}^{\infty}$ be a countable orthonormal basis of $H$. If we have

$$
\lim _{j \rightarrow \infty}\left\|T_{j} F_{n}\right\|=0
$$

for all $n$, then we have, for any $F \in H$,

$$
\lim _{j \rightarrow \infty}\left\|T_{j} F\right\|=0 \text {. }
$$

For each $f \in L^{2}\left(G_{k} \mid G_{A} / K\right)$, set

$$
T_{j} f=f * \xi_{j} / \operatorname{deg} \xi_{j}-(f, 1) / v .
$$

Then the mapping $f \mapsto T_{j} f$ gives rise to a bounded linear operator on $L^{2}\left(G_{k} \mid G_{A} / K\right)$. For any $x \in G_{A}$ and $f \in L^{2}\left(G_{k} \mid G_{A}\right)$, we set $R_{x} f(g)=$ $f(g x)$. Then $R_{x}$ is a norm preserving linear operator on $L^{2}\left(G_{k} \mid G_{A}\right)$. Since we can write, for any $f \in L^{2}\left(G_{k} \mid G_{A} / K\right)$,

$$
f * \xi_{j}=\sum_{l=1}^{\operatorname{deg} \xi_{j}} R_{\sigma_{l}^{(j)-1}} f
$$

we have

$$
\left\|f * \xi_{j}\right\| \leqq \sum_{l=1}^{\operatorname{deg} \xi_{j}}\left\|R_{\sigma_{l}^{(j)-1}} f\right\|=\operatorname{deg} \xi_{j}\|f\|
$$

Hence 


$$
\left\|T_{j} f\right\| \leqq\|f\|+\frac{|(f, 1)|}{v}\|1\| \leqq 2\|f\| .
$$

This implies that

$$
\sup _{j \geqq 1}\left\|T_{j}\right\| \leqq 2
$$

We shall pick up a well-chosen orthonormal basis of $L^{2}\left(G_{k} \mid G_{A} / K\right)$ and prove that the equality (2.1) holds for each member of this basis. Then Lemma 2 implies that the equality (2.1) will hold for any $f \in L^{2}\left(G_{k} \mid G_{A} / K\right)$ and hence Proposition 2 will be proved.

Let $\Pi$ be the right regular representation of $G_{A}$ on $L^{2}\left(G_{k} \mid G_{A}\right)$. Since $G_{k} \mid G_{A}$ is compact, the unitary representation $\Pi$ decomposes into a direct sum of at most countable irreducible unitary representations of $G_{A}$ with finite multiplicities (cf. [4], Chap. I, §2.3, Theorem). We write

$$
L^{2}\left(G_{k} \mid G_{A}\right)=\sum_{n=0}^{\infty} H^{(n)}, \quad \Pi=\sum_{n=0}^{\infty} \pi^{(n)}
$$

where $H^{(n)}$ is a closed $G_{A}$-invariant subspace of $L^{2}\left(G_{k} \mid G_{A}\right)$, and $\pi^{(n)}$ is the restriction of $\pi$ to $H^{(n)}\left(\pi^{(n)}\right.$ is irreducible). We may assume that $H^{(0)}=C \cdot 1$ and that $\pi^{(0)}$ is trivial. Furthermore each representation $\pi^{(n)}$ is factorizable. That is to say, there exists an irreducible unitary representation $\pi_{v}^{(n)}$ of $G_{k_{v}}$ for every place $v$ of $k$ satisfying the following conditions (2.2) and (2.3).

(2.2) Except for a finite number of $v$, the representation space $H_{v}^{(n)}$ of $\pi_{v}^{(n)}$ contains a $G_{o_{v}}$-invariant unit vector $f_{0}^{v}$ which is unique up to a scalar multiple.

(2.3) The restricted tensor product $\otimes_{v} \pi_{v}^{(n)}$ with respect to the family $\left\{f_{0}^{v}\right\}$ is unitarily equivalent to $\pi^{(n)}$ (cf. [4], Chap. III, $\S 6.2$ ).

Then we have the decomposition;

$$
L^{2}\left(G_{k} \mid G_{A} / K\right)=\sum_{n=0}^{\infty}\left(L^{2}\left(G_{k} \mid G_{A} / K\right) \cap H^{(n)}\right) .
$$

Now we shall choose an orthonormal basis of $L^{2}\left(G_{l_{k}} \mid G_{A} / K\right) \cap H^{(n)}$ for each $n$. Then the union of bases for all $n$ forms an orthonormal basis of $L^{2}\left(G_{k} \mid G_{A} / K\right)$. When $n=0$, we have

$$
L^{2}\left(G_{k} \mid G_{A} / K\right) \cap H^{(0)}=H^{(0)},
$$

and $\{1 / \sqrt{v}\}$ can be taken as its orthonormal basis. It is obvious that

$$
T_{j}(1 / \sqrt{v})=1 / \sqrt{v}-(1 / \sqrt{v}, 1) / v=0
$$


From now on, we fix an index $n \geqq 1$, and, for simplicity, we drop the index $n$. Hence we write $H, \pi, \pi_{v}$, and $H_{v}$ for $H^{(n)}, \pi^{(n)}, \pi_{v}^{(n)}$, and $H_{v}^{(n)}$ respectively. Note that, for any $F \in H \cap L^{2}\left(G_{k} \mid G_{A} / K\right)$, we have $(F, 1)=0$ and $T_{j} F=F * \xi_{j} / \operatorname{deg} \xi_{j}$. Let us take an isometric linear mapping $T$ from the restricted tensor product $\boldsymbol{\otimes}_{v} H_{v}$ to $H$, intertwining $\boldsymbol{\otimes}_{v} \pi_{v}$ and $\pi$. For $\mathfrak{g} \in \mathscr{P}_{f}$, let $V_{\mathfrak{g}}$ be the space of $K_{\mathfrak{g}}$ invariant vectors in $H_{8}$. Then $V_{\mathfrak{g}}$ is finite dimensional (cf. [1], Theorem 1). In view of (2.2), there exists a finite subset $\mathscr{S}^{\prime}$ of $\mathscr{P}_{f}$ containing $\mathscr{S}$ such that $V_{\mathrm{g}}$ is one dimensional if $\mathfrak{g} \in \mathscr{P}_{f}-\mathscr{S}^{\prime}$. Then we can take as a countable orthonormal basis of $H \cap L^{2}\left(G_{k} \mid G_{A}\right)$ $K)$ a set of elements of the form $T\left(\otimes \varphi^{v}\right)$ where $\varphi^{v} \in H_{v}$ for every place $v$ and satisfies the following condition (C):

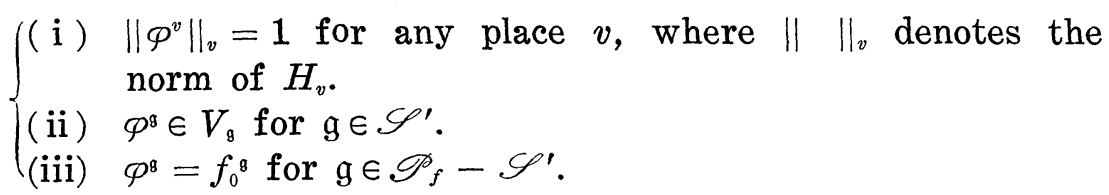

Hence, to show Proposition 2, it is enough to establish the following:

Proposition 3. Notation being as above, for any element $F \in H$ of the form $T\left(\bigotimes_{v} \varphi_{F}^{v}\right)$ where $\varphi_{F}^{v}$ satisfies the condition (C), we have

$$
\lim _{j \rightarrow \infty}\left\|F * \xi_{j} / \operatorname{deg} \xi_{j}\right\|=0 .
$$

Proof. We set $S_{\mathrm{g}}(j)=K_{\mathrm{g}}$ for all $j$ if $g \in \mathscr{P}_{f}-\mathscr{S}$. Let $\xi_{j}^{\mathrm{g}}$ be the characteristic function of $S_{8}(j)$ for $\mathfrak{g} \in \mathscr{P}_{f}$. Then $\xi_{j}{ }^{8}$ is $K_{\mathrm{g}}$-biinvariant, continuous, and compactly supported on $G_{k_{g}}$. For $g_{f}=$ $\left(\cdots, g_{\mathrm{g}}, \cdots\right) \in G_{A_{f}}$, we have

$$
\xi_{j}\left(g_{f}\right)=\prod_{g \in \mathscr{\sim}_{f}} \xi_{j}^{\mathrm{g}}\left(g_{\mathfrak{g}}\right) \text {. }
$$

For $g \in \mathscr{S}^{\prime}$, choose an orthonormal basis $\left\{f_{l}^{8} \mid 1 \leqq l \leqq \operatorname{dim} V_{g}\right\}$ of $V_{8}$. We may assume that $f_{1}^{g}=\varphi_{F}^{g}$. Then, for $\mathfrak{g} \in \mathscr{S}^{\prime}$, the integral

$$
f_{l}^{\mathrm{g} * \xi_{j}{ }^{\mathrm{g}}}=\int_{G_{k_{\mathrm{g}}}} \xi_{j}{ }^{\mathrm{g}}\left(g_{\mathrm{g}}^{-1}\right) \pi_{\mathrm{g}}\left(g_{\mathrm{g}}\right) f_{l}^{\mathrm{g}} d g_{\mathrm{g}}
$$

belongs to $H_{\mathfrak{g}}$ and is invariant under the action of $K_{\mathfrak{g}}$ through $\pi_{\mathrm{g}}$. Thus it belongs to $V_{\mathrm{g}}$ and hence can be written as a $C$-linear combination of $f_{m}^{\mathrm{a}}\left(1 \leqq m \leqq \operatorname{dim} V_{\mathrm{g}}\right)$. That is to say, we have

$$
f_{l}^{8} * \xi_{j}^{8}=\sum_{m=1}^{\operatorname{dim} V_{8}^{8}} f_{m}^{8} \cdot \lambda_{m, l}^{8}\left(\xi_{j}\right)
$$

where 


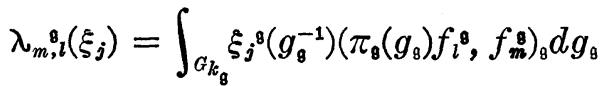

$$
\begin{aligned}
& =\int_{S_{8}(j)}\left(\pi_{\mathrm{g}}\left(g_{\mathrm{g}}^{-1}\right) f_{l}^{\mathrm{g}}, f_{m}^{\mathbf{g}}\right)_{8} d g_{\mathrm{g}} .
\end{aligned}
$$

(Here $(,)_{8}$ denotes the inner product of $H_{8}$.) Now we have, for any $x \in G_{A}$,

$$
\begin{aligned}
& F * \xi_{j}(x)=\int_{G_{A_{f}}} F\left(x g_{f}\right) \xi_{j}\left(g_{f}^{-1}\right) d g_{f} \\
& =\int_{a_{\boldsymbol{A}_{f}}}\left(\pi\left(g_{f}\right) T\left(\otimes \varphi_{F}^{v}\right)\right)(x) \xi_{j}\left(g_{f}^{-1}\right) d g_{f} \\
& =T\left(\bigotimes_{v \in \mathscr{G}_{\infty}} \varphi_{F}^{v} \otimes \bigotimes_{\mathrm{g} \in \mathscr{G}_{f} f} \int_{a_{k_{\mathrm{g}}}} \xi_{j}^{\mathrm{g}}\left(g_{\mathrm{g}}^{-1}\right) \pi_{\mathrm{g}}\left(g_{\mathrm{g}}\right) \varphi_{F}^{\mathrm{g}} d g_{\mathrm{g}}\right)(x)
\end{aligned}
$$

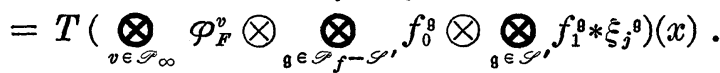

Since $T$ is norm-preserving, we get

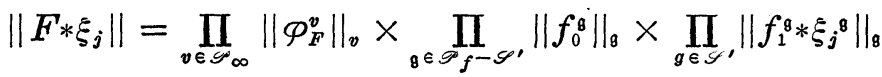

$$
\begin{aligned}
& =\prod_{\mathfrak{g} \in \mathscr{S}^{\prime}}\left\|\sum_{m=1}^{\mathrm{dim} V_{\mathfrak{g}}^{\mathfrak{g}}} f_{m}^{\mathfrak{g}} \cdot \lambda_{m, 1}^{\mathfrak{g}}\left(\xi_{j}\right)\right\|_{\mathbb{B}} \\
& \leqq \prod_{\mathbb{g} \in \mathscr{S}_{0}}\left(\sum_{m=1}^{\operatorname{dim} \boldsymbol{\nabla}_{\mathbb{8}}}\left|\lambda_{m, 1}^{\mathrm{g}}\left(\xi_{j}\right)\right|\right) \text {. }
\end{aligned}
$$

On the other hand, if we put

$$
\operatorname{deg} \xi_{j}{ }^{\mathrm{g}}=\int_{G_{k_{\mathrm{g}}}} \xi_{j}{ }^{\mathrm{g}}\left(g_{\mathrm{g}}\right) d g_{\mathrm{g}}=\int_{S_{\mathrm{g}}(j)} d g_{\mathrm{g}}
$$

for $\mathfrak{g} \in \mathscr{P}_{f}$, it is easy to see (recall the definition (1.2))

$$
\operatorname{deg} \xi_{j}=\prod_{g \in S^{\prime}} \operatorname{deg} \xi_{j}^{8}
$$

(in fact it equals $\Pi_{8 \in \mathscr{S}} \operatorname{deg} \xi_{j}{ }^{8}$ ). Thus we have

$$
\left\|F * \xi_{j} / \operatorname{deg} \xi_{j}\right\| \leqq \prod_{8 \in S^{\prime}}\left(\sum_{m=1}^{\mathrm{dim} \nabla_{\mathrm{g}}}\left|\lambda_{m, 1}^{\mathrm{g}}\left(\xi_{j}\right)\right| / \operatorname{deg} \xi_{j}^{8}\right) .
$$

Since $S_{\mathfrak{g}}(j)=K_{\mathfrak{g}}$ for $\mathfrak{g} \in \mathscr{S}^{\prime}-\mathscr{S}$, we get finally

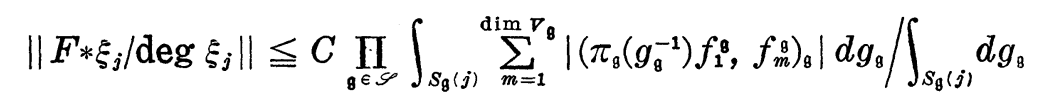

where $C=\Pi_{\mathrm{s} \in \mathscr{S}^{\prime}-\mathscr{S}} \operatorname{dim} V_{\mathrm{g}}$.

Now we shall show that $\pi_{\mathrm{g}}$ is not one dimensional if $\mathfrak{g} \in \mathscr{S}$ and $G_{k_{8}}$ is noncompact. More generally, we shall prove the following:

LEMMa 3. Let $H$ be a connected, simply connected semisimple 
linear algebraic group defined over an algebraic number field $k$, and let $v$ be a place of $k$. Assume that $H$ is $k_{v}$-almost simple and that $H_{k} H_{k_{v}}$ is dense in $H_{A}$. Let $\rho$ be an irreducible unitary representation of $H_{A}$ realized on a closed subspace $\mathscr{H}$ of $L^{2}\left(H_{k} \backslash H_{A}\right)$ by right translation. Furthermore assume that $\rho$ decomposes into a restricted tensor product $\otimes \rho_{w}$ of irreducible unitary representations $\rho_{w}$ of $H_{k_{w}}$. If $\rho$ is nontrivial, then $\rho_{v}$ is not one dimensional.

Proof. Since $H_{k} H_{k_{v}}$ is dense in $H_{A}, H_{k_{v}}$ is not compact, and hence $\operatorname{rank}_{k_{v}} H \geqq 1$ (cf. [9], p. 187). It is known that, if $X$ is a semisimple, simply connected, almost simple linear algebraic group defined over a local field $K$ with positive $K$-rank, then $X_{K}$ coincides with its own commutator (cf. [3], 6-4 and 6-15; see also [6], Appendix II, Theorem). Hence $H_{k_{v}}$ has no nontrivial unitary characters. Assume that $\rho_{v}$ is trivial. Then every element in $\mathscr{H}$ is right $H_{k_{v}}$-invariant as a function on $H_{A}$. There exists $\varphi \in C_{c}^{0}\left(H_{A}\right)$ and $f \in \mathscr{H}$ such that

$$
\left.F=\int_{H_{A}} \varphi(h) \rho(h) f d h \neq 0 \text { (as an element of } \mathscr{H}\right) \text {. }
$$

It is easy to see that $F$ is, as a function on $H_{A}$, continuous. Since $F$ is right $H_{k_{v}}$-invariant and left $H_{k}$-invariant, $F$ is constant on $H_{k} H_{k_{v}}$ which is dense in $H_{A}$. Hence $F$ is a nonzero constant function on $H_{A}$. Since $\rho$ is irreducible, we have $\mathscr{H}=C \cdot 1$ and $\rho$ is trivial. Contradiction! The lemma is proved.

Applying Lemma 3 for $(H, \rho, v)=(G, \pi, \mathfrak{g})\left(\mathfrak{g} \in \mathscr{S}\right.$ and $G_{k_{\mathfrak{g}}}$ is noncompact), we see that $\pi_{8}$ is not one dimensional.

On the other hand, Howe and Moore proved the following:

Lemma 4 (cf. [6] Theorem 5.2). ${ }^{6)}$ Let $\pi$ be an irreducible unitary representation of $k$-almost simple, simply connected linear algebraic group 'G defined over a local field $k$ on a Hilbert space $H$. For any $x, y \in H$, set $\rho_{x, y}(g)=(\pi(g) x, y)$. Then $\rho_{x, y}$ vanishes at infinity if $\pi$ is not one dimensional.

(Here we say that a continuous function $f$ on a locally compact group $G$ vanishes at infinity, if, for any $\varepsilon>0$, there exists a compact subset $C$ of $G$ such that $\sup _{g \in G-C}|f(g)|<\varepsilon$. In case that $G$ is

6) In case $G=\mathrm{SL}(2)$ over a $g$-adic field $k_{g}$, this result easily follows from the existence of the Kirillov model of $\rho$ (that is to say, $\rho$ is realized on a closed subspace $\mathscr{C}$ of $L^{2}\left(k_{g}^{\mathrm{x}}\right)$, and, for any $f \in \mathscr{H}$ and for any $a \in k_{g}^{\mathrm{x}}$, we have

$$
\rho\left(\begin{array}{cc}
a & \\
& a^{-1}
\end{array}\right) f(x)=f\left(a^{2} x\right) \quad\left(x \in k_{g}^{\mathrm{x}}\right)
$$

(cf. [4], Appendix to Chapter II, $n^{\circ} 2$ and $\left.n^{\circ} 5\right)$ ). 
compact, every continuous function is said to vanish at infinity.)

Thus we conclude that the function on $G_{k_{\mathrm{g}}}$ given by $g_{\mathrm{g}} \mapsto$ $\left|\left(\pi_{\mathrm{g}}\left(g_{\mathrm{g}}^{-1}\right) f_{1}^{\mathrm{g}}, f_{m}^{\mathfrak{a}}\right)_{\mathbb{g}}\right|\left(1 \leqq m \leqq \operatorname{dim} V_{\mathrm{g}}\right)$ vanishes at infinity. In view of (2.6), the proof of Proposition 3 has now been reduced to the following lemma.

LEMMA 5. Let $G_{l}(1 \leqq l \leqq m)$ be a locally compact group with a left invariant measure $d g_{l}$. Let $f_{l}(1 \leqq l \leqq m)$ be a continuous function on $G_{l}$ vanishing at infinity. Let $\left\{S_{l}(j)\right\}_{j=1}^{\infty}$ be a sequence of open compact subsets of $G_{l}$ such that

$$
\inf _{j \geqq 1} \int_{S_{l^{(j)}}} d g_{l}=\eta_{l}>0 \quad(1 \leqq l \leqq m)
$$

If

$$
\lim _{j \rightarrow \infty} \prod_{l=1}^{m} \int_{S_{l}(j)} d g_{l}=+\infty,
$$

then the following equality holds:

$$
\lim _{j \rightarrow \infty} \prod_{l=1}^{m}\left(\int_{S_{l}(j)} f_{l}\left(g_{l}\right) d g_{l} / \int_{S_{l}(j)} d g_{l}\right)=0
$$

Proof. Note that (2.7) implies that, for at least one $l \in M=$ $\{1,2, \cdots, m\}, G_{l}$ is noncompact. Observe that

$$
\left|\int_{s_{l}(j)} f_{l}\left(g_{l}\right) d g_{l} / \int_{s_{l}(j)} d g_{l}\right|
$$

is bounded if $G_{l}$ is compact. Hence we may assume that $G_{l}$ is noncompact for every $l \in M$. Set $N_{l}=\sup _{g \in G_{l}}\left|f_{l}(g)\right|$. Then $N_{l}<\infty(1 \leqq$ $l \leqq m)$. For any $\varepsilon>0$, there is a compact subset $C_{l}$ of $G_{l}$ such that $\left|f_{l}(g)\right|<\varepsilon$ for every $g \in G_{l}-C_{l}$. By a simple calculation, we have

$$
\begin{aligned}
& \prod_{l \in M} \int_{S_{l}(j)} f_{l}\left(g_{l}\right) d g_{l} \\
& \quad=\sum_{\Lambda \subset M} \prod_{l \in \Lambda} \int_{S_{\left.l^{(j)}\right) \cap c_{l}}} f_{l}\left(g_{l}\right) d g_{l} \\
& \quad \cdot \prod_{l \in M-\Lambda} \int_{S_{l}(j) \cap\left(G_{l}-C_{l}\right)} f_{l}\left(g_{l}\right) d g_{l}
\end{aligned}
$$

where $A$ ranges over the collection of all subsets of $M$. To simplify the notation, we set

Since

$$
J_{l}=\int_{c_{l}} d g_{l} \text { and } K_{l}(j)=\int_{S_{l}(j)} d g_{l} .
$$




$$
\left|\int_{S_{l}(j) \cap c_{l}} f_{l}\left(g_{l}\right) d g_{l}\right| \leqq \operatorname{Min}\left(N_{l} J_{l}, N_{l} K_{l}(j)\right)
$$

and

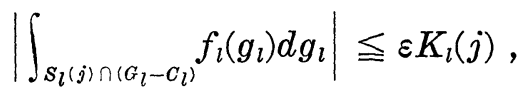

we have

$$
\begin{aligned}
\left|\prod_{l \in M}\left\{\int_{S_{l}(j)} f_{l}\left(g_{l}\right) d g_{l} / K_{l}(j)\right\}\right| \\
\quad \leqq \prod_{l \in M} N_{l} J_{l} / \prod_{l \in M} S_{l}(j) \\
\quad+\sum_{l \in M} \prod_{l \in A} N_{l} K_{l}(j) \prod_{l \in M-A} \varepsilon \cdot K_{l}(j) \cdot \prod_{l \in M} K_{l}(j)^{-1} \\
\quad=\prod_{l \in M} N_{l} J_{l} / \prod_{l \in M} S_{l}(j)+\sum_{\lambda \in M} \varepsilon^{|M-\Lambda|} \cdot \prod_{l \in \Lambda} N_{l} .
\end{aligned}
$$

Here $|M-\Lambda|$ denotes the number of elements in the set $M-\Lambda$. Hence, if $\varepsilon<1$, we have

$$
\begin{aligned}
\limsup _{j \rightarrow \infty} & \left|\prod_{l \in M}\left\{\int_{S_{l}(j)} f_{l}\left(g_{l}\right) d g_{l} / K_{l}(j)\right\}\right| \\
& \leqq \varepsilon \sum_{\lambda \subsetneq M} \prod_{l \in A} N_{l} .
\end{aligned}
$$

Since we can choose arbitrary small $\varepsilon>0$, we obtain the equality (2.8).

Thus Theorem 1 has been established.

3. In this section, we always assume $G=\mathrm{SL}_{2}$ (regarded as a linear algebraic group defined over an algebraic number field $k$ ). We shall prove Proposition 2 under our assumptions. Note that Proposition 2 for $G=\mathrm{SL}_{2}$ implies Theorem 2, by virtue of Proposition 1. We set

$$
U=\left\{\left(\begin{array}{rr}
1 & u \\
& 1
\end{array}\right)\right\}, \quad H=\left\{\left(\begin{array}{cc}
t & \\
& t^{-1}
\end{array}\right) \mid t \neq 0\right\}, \quad \text { and } P=U H .
$$

These groups can be naturally regarded as $k$-subgroups of $G$. For any $F \in C_{c}^{0}\left(U_{A} H_{k} \mid G_{A}\right)$, set

$$
\theta_{F}(g)=\sum_{\gamma \in P_{k} \backslash G_{k}} F(\gamma g) .
$$

The series (3.1) converges absolutely and uniformly on any compact subset of $G_{A}$. The function $\theta_{F}$ is, as a function on $G_{k} \mid G_{A}$, continuous and compactly supported, and hence square integrable on $G_{k} \mid G_{A}$ (cf. [5], §6). Let $\Theta$ be the closure of the subspace of $L^{2}\left(G_{k} \mid G_{A}\right)$ spanned by all elements of the form $\theta_{F}$ with $F \in C_{c}^{0}\left(U_{A} H_{k} \mid G_{A}\right)$. Let $\mathscr{H}$ be 
the closed subspace of $L^{2}\left(G_{k} \mid G_{A}\right)$ consisting of all elements $f$ such that the integral $\int_{U_{k} \backslash U_{A}} f(u g) d u$ vanishes for almost all $g \in G_{A}$. Then $\Theta$ and $\mathscr{H}$ are both right $G_{A}$-invariant. It is known that $L^{2}\left(G_{k} \mid G_{A}\right)$ is the direct orthogonal sum of $\Theta$ and $\mathscr{C}$ (cf. [5], §7). It follows that $L^{2}\left(G_{k} \mid G_{A} / K\right)$ is the direct orthogonal sum of $\widetilde{\Theta}=\Theta \cap L^{2}\left(G_{k} \mid G_{A} / K\right)$ and $\widetilde{\mathscr{H}}=\mathscr{H} \cap L^{2}\left(G_{k} \mid G_{A} / K\right) ; L^{2}\left(G_{k} \mid G_{A} / K\right)=\widetilde{\Theta} \oplus \tilde{\mathscr{H}}$ (direct orthogonal sum). Hence, for any $f \in L^{2}\left(G_{k} \mid G_{A} / K\right)$, we can write $f=\varphi+\psi$ where $\varphi \in \widetilde{\Theta}$ and $\psi \in \widetilde{\mathscr{H}}$. As is well-known, $\widetilde{\Theta}$ contains constant functions. Hence $\tilde{\mathscr{H}}$ is orthogonal to $\boldsymbol{C} \cdot 1$. Thus we have

$$
\left\|f * \xi_{j} / \operatorname{deg} \xi_{j}-(f, 1) / v\right\| \leqq\left\|\varphi * \xi_{j} / \operatorname{deg} \xi_{j}-(\varphi, 1) / v\right\|+\left\|\psi * \xi_{j} / \operatorname{deg} \xi_{j}\right\| .
$$

Hence the proof of Proposition 2 in our case has now been reduced to the verification of the following two propositions.

Proposition 4. If (0.1) is satisfied, the equality

$$
\lim _{j \rightarrow \infty}\left\|\varphi * \xi_{j} / \operatorname{deg} \xi_{j}-(\varphi, 1) / v\right\|=0
$$

holds for any $\varphi \in \widetilde{\Theta}$.

Proposition 5. If (0.1) is satisfied, the equality

$$
\lim _{j \rightarrow \infty}\left\|\psi * \xi_{j} / \operatorname{deg} \xi_{j}\right\|=0
$$

holds for any $\psi \in \tilde{\mathscr{H}}$.

It is known that the right regular representation on $\mathscr{H}$ decomposes into a direct orthogonal sum of at most countable irreducible and factorizable unitary representations with finite multiplicities (cf. [5], 22 and [4], Chap. III, §3-3, Theorem). Then, to prove Proposition 5, we just repeat the argument of the proof of Proposition 2 in $\S 2$, replacing $L^{2}\left(G_{k} \mid G_{A} / K\right)$ with $\tilde{\mathscr{H}}$.

In order to show Proposition 4, we need several results about the spectral decomposition of $\Theta$.

Let $M=\Pi_{v} M_{v}$ be the maximal compact subgroup of $G_{A}$, where we set

$$
M_{v}= \begin{cases}G_{O_{v}} & \text { if } v \in \mathscr{P}_{f} \\ S O(2) \text { if } v \in \mathscr{P}_{\infty} \text { and } k_{v} \cong \boldsymbol{R} \\ S U(2) \text { if } v \in \mathscr{P}_{\infty} \text { and } k_{v} \cong \boldsymbol{C} .\end{cases}
$$

Then we have $G_{A}=U_{A} H_{A} M$. We fix, once and for all, the Iwasawa 
decomposition of $g \in G_{A}$ given by

$$
g=\underline{u}(g) \underline{h}(g) \underline{m}(g),
$$

where $\underline{u}(g) \in U_{A}, \underline{h}(g) \in H_{A}$, and $\underline{m}(g) \in M$. We normalize the Haar measure $d g$ on $G_{A}$ by putting, for any $f \in C_{c}^{0}\left(G_{A}\right)$,

$$
\int_{G_{\boldsymbol{A}}} f(g) d g=\int_{M} d m \int_{H_{\boldsymbol{A}}}|\beta(h)|_{\boldsymbol{A}}^{-1} d h \int_{U_{\boldsymbol{A}}} f(u h m) d u .
$$

Here we set

$$
\beta(h)=t^{2}
$$

for

$$
h=\left(\begin{array}{cc}
t & 0 \\
0 & t^{-1}
\end{array}\right) \in H_{A},
$$

and we denote by $d u, d h$, and $d m$ Haar measures on $U_{A}, H_{A}$, and $M$ respectively, which are normalized by the following conditions;

$$
\int_{U_{k} \backslash U_{\boldsymbol{\Delta}}} d u=1, \int_{H_{k} \backslash H_{\boldsymbol{A}},|\beta(h)| \leqq 1}|\beta(h)|_{A}^{s} d h=1 / s \quad(\operatorname{Re} s>0),
$$

and

$$
\int_{M} d m=1
$$

From now on, we normalize the Haar measure $d g_{\mathrm{g}}$ on $G_{k_{\mathrm{g}}}\left(\mathfrak{g} \in \mathscr{P}_{f}\right)$ so that $\int_{M_{g}} d g_{8}=1$. Let $d g_{f}$ be a Haar measure on $G_{A_{f}}$ given by

$$
d g_{f}=\prod_{\mathfrak{s} \in \mathscr{O}_{f}} d g_{\mathfrak{s}} \quad\left(g_{f}=\prod_{\mathfrak{s} \in \mathscr{O}_{f}} g_{\mathfrak{s}} \in G_{A_{f}}\right) .
$$

We normalize the Haar measure $d g_{\infty}$ on $G_{\infty}$ so that $d g=d g_{\infty} d g_{f}$ $\left(g=g_{\infty} g_{f}\right)$, where $d g$ and $d g_{f}$ are given by (3.4) and (3.5), respectively.

Let $I_{1}$ be the subgroup of $I$ consisting of ideles with module 1 . For a positive real number $\lambda$, we denote by $\xi(\lambda)$ the idele such that $\xi(\lambda)_{\mathfrak{s}}=1$ for every $\mathfrak{g} \in \mathscr{P}_{f}$ and $\xi(\lambda)_{v}=\lambda$ for every $v \in \mathscr{P}_{\infty}$. Let $N$ be the image of $\{\xi(\lambda) ; \lambda>0\}$ by the natural projection from $I$ to $k^{\mathrm{x}} \backslash \boldsymbol{I}$. Then we have

$$
k^{\mathrm{x}} \backslash \boldsymbol{I}=\left(\boldsymbol{k}^{\mathrm{x}} \backslash \boldsymbol{I}_{1}\right) \times N \quad \text { (direct product) } .
$$

Let $X_{1}$ be the set of all unitary characters on $H_{k} \backslash H_{A}$ which are trivial on the image of $N$ by the natural isomorphism from $k^{\mathrm{x}} \backslash I$ to $H_{k} \backslash H_{A}$. Then $X_{1}$ can be identified with the dual of $k^{\mathrm{x}} \backslash I_{1}$. Since $k^{\mathrm{x}} \backslash I_{1}$ is compact, $X_{1}$ is discrete (cf. [15], Chap. VII, $\S 4$ ). 
We fix a complete system $M^{\wedge}$ of representatives of equivalence classes of finite dimensional irreducible unitary representations of $M$. Let $H_{\tau}$ be the representation space of $\tau \in M^{\wedge}$. For $\chi \in X_{1}$, let $H_{\tau}(\chi)$ be the subspace of $H_{\tau}$ consisting of all vectors $v \in H_{\tau}$ which satisfy the following equality:

$$
v \cdot \tau(u h)=v \cdot \chi^{-1}(h) \quad\left(\forall u h \in P_{A} \cap M\right) .
$$

We denote by $X_{1}(\tau)$ the set of all elements $\chi \in X_{1}$ such that $H_{\tau}(\chi) \neq$ 0 . It is easy to see that $X_{1}(\tau)$ is a finite set.

For $F \in C_{c}^{0}\left(U_{A} H_{k} \mid G_{A}\right), \tau \in M^{\wedge}, \chi \in X_{1}(\tau)$, and for $s \in C$, we set

$$
F^{\wedge}(s, \chi, \tau)=\int_{M} \int_{H_{k^{\prime} / H_{A}}} F(h m) \tau\left(m^{-1}\right) \chi(h)|\beta(h)|_{A}^{-s} d h d m .
$$

The integral (3.6) converges for any $s \in C$. As a function of $s$, $F^{\wedge}(s, \chi, \tau)$ is a holomorphic function in $C$ with values in $\operatorname{End}_{c}\left(H_{\tau}\right)$. Set

$$
\theta_{F}(s, \chi, \tau)=\int_{M} \int_{H_{k} \backslash H_{A}} \int_{U_{k} \backslash U_{A}} \theta_{F}(u h m) \tau\left(m^{-1}\right) \chi(h)|\beta(h)|_{A}^{s-1} d u d h d m,
$$

where $\theta_{F}$ is given by (3.1). The integral (3.7) converges absolutely and uniformly on any compact subset of the domain $\{s \in C \mid \operatorname{Re} s>1\}$. As a function of $s, \theta_{F}(s, \chi, \tau)$ is continued to a meromorphic function in $C$ with values in $\operatorname{End}_{c}\left(H_{\tau}\right)$ (cf. [5], §6). It is known that

$$
\theta_{F}^{\wedge}(s, \chi, \tau)=F^{\wedge}(1-s, \chi, \tau)+F^{\wedge}\left(s, \chi^{-1}, \tau\right) \Phi(s ; \chi, \tau),
$$

where $\Phi(s ; \chi, \tau)$ is a meromorphic function of $s$ in $C$ with values in $\operatorname{End}_{c}\left(H_{\tau}\right)$ (cf. [5], §6). Furthermore suppose that, as a function on $G_{A}, F(g)$ depends smoothly with respect to the archimedean components of $g$. Then the norm of $\theta_{F}$ in $L^{2}\left(G_{k} \mid G_{A}\right)$ is given by the following formula (cf. [5], §7, (7.8)):

$$
\left\|\theta_{F}\right\|^{2}=\frac{1}{2 \pi \sqrt{-1}} \sum_{\tau \in M^{\wedge}} \sum_{\chi \in X_{1}(\tau)} \int_{J}\left\|\theta_{F}^{\widehat{F}}(\varepsilon, \chi, \tau)\right\|_{\tau}^{2} d s+\frac{1}{v}\left|\left(\theta_{F}, 1\right)\right|^{2} .
$$

Here $\|T\|_{\tau}^{2}$ denotes $\operatorname{dim} \tau \cdot \operatorname{Tr}\left(T T^{*}\right)$ for $T \in \operatorname{End}_{c}\left(H_{\tau}\right)$, and we set

$$
J=\left\{s \in C \mid \operatorname{Re} s=\frac{1}{2}, \operatorname{Im} s<0\right\} .
$$

The following lemma is easily proved.

LEMma 6. Let $\left\{T_{j}\right\}_{j=1}^{\infty}$ be a sequence of bounded linear operators on a Hilbert space $H$ such that $\sup _{j \geq 1}\left\|T_{j}\right\|<\infty$, and let $H^{\prime}$ be a dense subspace of $H$. Assume that, for any $v \in H^{\prime}$, 


$$
\lim _{j \rightarrow \infty}\left\|T_{j} v\right\|=0 .
$$

Then the equality (3.11) holds for any $v \in H$.

Now we are ready to prove Proposition 4 . Let $T_{j}$ be a linear operator on $\widetilde{\Theta}$ given by

$$
T_{j} \varphi=\varphi * \xi_{j} / \operatorname{deg} \xi_{j}-(\varphi, 1) / v \quad(\varphi \in \widetilde{\Theta}) .
$$

We have already seen that $\left\|T_{j}\right\| \leqq 2(j=1,2, \cdots)$. We set $M_{\infty}=$ $\Pi_{v \in \mathscr{\vartheta}_{\infty}} M_{v}$. Then $M_{\infty}$ is a maximal compact subgroup of $G_{\infty}$. Let $\mathscr{D}$ be the space consisting of all continuous functions on $U_{A} H_{k} \mid G_{A} / K$ satisfying the following conditions (3.12) and (3.13).

(3.12) $F(g)$ is compactly supported modulo $U_{A} H_{k}$.

(3.13) As a function on $G_{A}, F(g)$ depends smoothly on $G_{\infty}$ and $F(g)$ is right $M_{\infty}$-finite.

Let $\Theta^{\prime}$ be the linear space spanned by elements $\theta_{F}$ with $F \in \mathscr{D}$. Then $\Theta^{\prime}$ is a dense subspace of $\widetilde{\Theta}$.

Now we shall prove that the following equality holds for any $\theta_{F} \in \Theta^{\prime}:$

$$
\lim _{j \rightarrow \infty}\left\|T_{j} \theta_{F}\right\|=0 .
$$

Then, in view of Lemma 6, Proposition 4 will be proved. To show the equality (3.14), we need the next lemma.

Lemma 7. For any $\theta_{F} \in \Theta^{\prime}$, we have

$$
\left\|T_{j} \theta_{F}\right\|^{2}=\frac{1}{2 \pi \sqrt{-1}} \sum_{\tau \in M^{\wedge}} \sum_{\chi \in X_{1}(\tau)} \int_{J}\left\|\left(\theta_{F} * \xi_{j}\right)^{\wedge}(s, \chi, \tau) / \operatorname{deg} \xi_{j}\right\|_{\tau}^{2} d s .
$$

Proof. We have

$$
\begin{aligned}
\left\|T_{j} \theta_{F}\right\|^{2}= & \left\|\theta_{F} * \xi_{j} / \operatorname{deg} \xi_{j}-\left(\theta_{F}, 1\right) / v\right\|^{2} \\
= & \left\|\theta_{F} * \xi_{j} / \operatorname{deg} \xi_{j}\right\|^{2}-2 \operatorname{Re}\left\{\left(\overline{\theta_{F}, 1}\right) v^{-1} / \operatorname{deg} \xi_{j} \cdot\left(\theta_{F} * \xi_{j}, 1\right)\right\} \\
& +\left\|\left(\theta_{F}, 1\right) / v\right\|^{2} .
\end{aligned}
$$

We set $\xi_{j}(g)=\xi_{j}\left(g^{-1}\right)$. Then it is easily verified that

$$
\left(f_{1} * \xi_{j}, f_{2}\right)=\left(f_{1}, f_{2} * \xi_{j}^{\sim}\right) \quad\left(f_{1}, f_{2} \in L^{2}\left(G_{k} \mid G_{A} / K\right)\right),
$$

and that $\operatorname{deg} \xi_{j}=\operatorname{deg} \xi_{j}^{\curlyvee}$. Hence we have

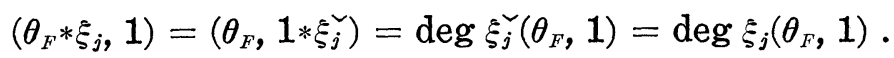

Thus 


$$
\left\|T_{j} \theta_{F}\right\|^{2}=\left\|\theta_{F} * \xi_{j} / \mathrm{deg} \xi_{j}\right\|^{2}-\left|\left(\theta_{F}, 1\right)\right|^{2} / v \text {. }
$$

Observe that $\theta_{F} * \xi_{j}$ also belongs to $\Theta^{\prime}$. Applying the formula (3.9) to $\theta_{F} * \xi_{j}$, we have

$$
\begin{aligned}
\left\|T_{j} \theta_{F}\right\|^{2}= & \frac{1}{2 \pi \sqrt{-1}} \sum_{\tau \in M^{\wedge}} \sum_{\chi \in X_{1}(\tau)} \int_{J}\left\|\left(\theta_{F} * \xi_{j}\right)^{\wedge}(s, \chi, \tau) / \operatorname{deg} \xi_{j}\right\|_{\tau}^{2} d s \\
& +\frac{1}{v}\left|\left(\theta_{F} * \xi_{j} / \operatorname{deg} \xi_{j}, 1\right)\right|^{2}-\left|\left(\theta_{F}, 1\right)\right|^{2} / v .
\end{aligned}
$$

The equality (3.16) implies that the last two terms of the right side of the above equality cancel each other, and hence the lemma is proved.

Since $\theta_{F}$ is, as a function on $G_{\infty}$, right $M_{\infty}$-finite, and since $\theta_{F} * \xi_{j}$ is right $K$-invariant, there exists a finite subset $L$ of $M^{\wedge}$ such that $\tau \in M^{\wedge}-L$ always implies $\left(\theta_{F} * \xi_{j}\right)(s, \chi, \tau)=0(j=1,2, \cdots)$ for any $s \in C$ and for any $\chi \in X_{1}(\tau)$. Thus the right side of (3.15) is a finite sum. Hence, to verify the equality (3.14), we have only to show that the following equality holds for any $\tau \in M^{\wedge}$ and any $\chi \in \chi_{1}(\tau)$ :

$$
\lim _{j \rightarrow \infty} \frac{1}{2 \pi \sqrt{-1}} \int_{J}\left\|\left(\theta_{F} * \xi_{j}\right)^{\wedge}(s, \chi, \tau) / \operatorname{deg} \xi_{j}\right\|_{\tau}^{2} d s=0
$$

Observe that

$$
\begin{aligned}
& \frac{1}{2 \pi \sqrt{-1}} \int_{J}\left\|\left(\theta_{F} * \xi_{j}\right)^{\wedge}(s, \chi, \tau) / \operatorname{deg} \xi_{j}\right\|_{\tau}^{2} d s \\
& \leqq \frac{1}{2 \pi \sqrt{-1}} \int_{J} \frac{1}{|s|^{2}} d s \times \sup _{s \in J}\left\|s \cdot\left(\theta_{F} * \xi_{j}\right)^{\wedge}(s, \chi, \tau) / \operatorname{deg} \xi_{j}\right\|_{\tau}^{2} \\
& \quad=\frac{1}{2} \sup _{s \in J}\left\|s \cdot\left(\theta_{F} * \xi_{j}\right)^{\wedge}(s, \chi, \tau) / \operatorname{deg} \xi_{j}\right\|_{\tau}^{2} .
\end{aligned}
$$

Hence the proof of (3.17), and hence of Proposition 4 has now been reduced to the verification of the following equality for any $F \in \mathscr{D}$, $\tau \in M^{\wedge}$, and for any $\chi \in X_{1}(\tau)$ under the assumption (0.1):

$$
\lim _{j \rightarrow \infty}\left\{\sup _{s \in J}\left\|s \cdot\left(\theta_{F} * \xi_{j}\right)^{\wedge}(s, \chi, \tau) / \operatorname{deg} \xi_{j}\right\|_{\tau}\right\}=0
$$

(recall that $J$ is given by (3.10)).

To establish the equality (3.18), we need the following lemma.

Lemma 8. For $F \in \mathscr{D}, \tau \in M^{\wedge}$, and $\chi \in X_{1}(\tau)$, there exists a positive constant $C$ such that the following inequality holds for any $s \in J=\{s \in C \mid \operatorname{Re} s=1 / 2, \operatorname{Im} s<0\}:$ 


$$
\begin{gathered}
\left\|s \cdot\left(\theta_{F} * \xi_{j}\right)^{\wedge}(s, \chi, \tau)\right\|_{\tau} \leqq C \int_{M}\left(\int_{G_{A_{f}}}\left|\beta\left(\underline{h}\left(m g_{f}^{-1}\right)\right)\right|_{A}^{1 / 2} \xi_{j}\left(g_{f}\right) d g_{f} d m\right. \\
(j=1,2, \cdots) .
\end{gathered}
$$

Proof. We set

$$
F * \xi_{j}(g)=\int_{G_{A_{f}}} F\left(g h_{f}^{-1}\right) \xi_{j}\left(h_{f}\right) d h_{f} .
$$

Then it is easily verified that $F * \xi_{j}$ also belongs to $\mathscr{D}$, and that $\theta_{F} * \xi_{j}=\theta_{F^{*} j_{j}}$. Applying (3.8) to $\theta_{F} * \xi_{j}=\theta_{F^{*} \xi j}$, we obtain

$$
\begin{aligned}
&\left(\theta_{F} * \xi_{j}\right)^{\wedge}(s, \chi, \tau)=\left(F * \xi_{j}\right)^{\wedge}(1-s, \chi, \tau) \\
&+\left(F * \xi_{j}\right)^{\wedge}\left(s, \chi^{-1}, \tau\right) \Phi(s ; \chi, \tau) .
\end{aligned}
$$

In view of (3.6) and (3.20), we have

$$
\begin{aligned}
&\left(F * \xi_{j}\right)^{\wedge}(s, \chi, \tau) \\
& \quad=\int_{M} \int_{H_{k} \backslash H_{A}} \int_{{G_{A}}_{f}} F\left(h m g_{f}^{-1}\right) \xi_{j}\left(g_{f}\right) \tau\left(m^{-1}\right) \chi(h)|\beta(h)|_{A}^{-s} d g_{f} d h d m .
\end{aligned}
$$

Observing that

$$
h m g_{f}^{-1}=h \underline{u}\left(m g_{f}^{-1}\right) h^{-1} \cdot h \underline{h}\left(m g_{f}^{-1}\right) \cdot \underline{m}\left(m g_{f}^{-1}\right),
$$

we have

$$
\begin{aligned}
\left(F * \xi_{j}\right)^{\wedge}(s, \chi, \tau) & \\
= & \int_{M} \int_{H_{k} \backslash H_{A}} \int_{\theta_{A_{f}}} F\left(h \underline{h}\left(m g_{f}^{-1}\right) \underline{m}\left(m g_{f}^{-1}\right)\right) \xi_{j}\left(g_{f}\right) \tau\left(m^{-1}\right) \chi(h)|\beta(h)|_{A}^{-s} d g_{f} d h d m \\
= & \int_{M} \int_{G_{A f}}\left(\int_{H_{k} \backslash H_{A}} F\left(h \cdot \underline{m}\left(m g_{f}^{-1}\right)\right) \chi(h)|\beta(h)|_{A}^{-s} d h\right) \chi \chi^{-1}\left(\underline{h}\left(m g_{f}^{-1}\right)\right) \\
& \cdot\left|\beta\left(\underline{h}\left(m g_{f}^{-1}\right)\right)\right|_{A}^{s} \xi_{j}\left(g_{f}\right) \tau\left(m^{-1}\right) d g_{f} d m
\end{aligned}
$$

(note that $h \underline{u}\left(m g_{f}^{-1}\right) h^{-1} \in U_{A}$ and that $F$ is left $U_{A^{-}}$invariant). Set

$$
F^{\wedge}(g, s, \chi)=\int_{H_{k} \backslash H_{A}} F(h g) \chi(h)|\beta(h)|_{A}^{-8} d h .
$$

This integral converges absolutely for any $s \in C$ and for any $g \in G_{A}$. Then,

$$
\begin{gathered}
\left(F * \xi_{j}\right)^{\wedge}(s, \chi, \tau)=\int_{M} \int_{G_{A_{f}}} F^{\wedge}\left(\underline{m}\left(m g_{f}^{-1}\right), s, \chi\right) \chi^{-1}\left(\underline{h}\left(m g_{f}^{-1}\right)\right) \\
\cdot\left|\beta\left(\underline{h}\left(m g_{f}^{-1}\right)\right)\right|_{A}^{s} \xi_{j}\left(g_{f}\right) \tau\left(m^{-1}\right) d g_{f} d m .
\end{gathered}
$$

Observe that

$$
\int_{\underline{M}} \tau\left(m^{-1}\right) F^{\wedge}(m, s, \chi) d m=F^{\wedge}(s, \chi, \tau)
$$


Then applying Peter-Weyl's theorem, we have

$$
F^{\wedge}(m, s, \chi)=\sum_{\tau \in M^{\wedge}} \operatorname{dim} \tau \cdot \operatorname{Tr}\left[\tau(m) F^{\wedge}(s, \chi, \tau)\right]
$$

for $m \in M$. Since $F$ is, as a function on $G_{A}$, right $M$-finite, the right side of (3.23) is a finite sum. Moreover it is known that $F^{\wedge}(s, \chi, \tau)$ is, as a function of $s$, rapidly decreasing at infinity in any vertical strip (cf. [5], $\S 7$ ). Hence, if $P(s)$ is a polynomial of $s$, we have

$$
\sup _{\operatorname{Re} s=1 / 2, m \in M}\left|P(s) \cdot F^{\wedge}(m, s, \chi)\right|<\infty
$$

We set

$$
C_{1}=\sup _{s \in J}\left|s \cdot F^{\wedge}(m, 1-s, \chi)\right|
$$

and

$$
C_{2}=\sup _{s \in J \cdot m \in M}\left|s \cdot F^{\wedge}\left(m, s, \chi^{-1}\right)\right| .
$$

In view of (3.22), we have, for $s \in J=\{s \in C \mid \operatorname{Re} s=1 / 2, \operatorname{Im} s<0\}$,

$$
\begin{aligned}
&\left\|s \cdot\left(F * \xi_{j}\right)^{\wedge}(1-s, \chi, \tau)\right\|_{\tau} \leqq \int_{M} \int_{G_{A_{f}}}\left|s \cdot F^{\wedge}\left(\underline{m}\left(m g_{f}^{-1}\right), 1-s, \chi\right)\right| \\
& \quad \cdot\left|\beta\left(\underline{h}\left(m g_{f}^{-1}\right)\right)\right|_{A}^{1 / 2} \xi_{j}\left(g_{f}\right)\left\|\tau\left(m^{-1}\right)\right\|_{\tau} d g_{f} d m \\
& \leqq C_{1} \cdot \operatorname{dim} \tau \int_{M} \int_{G_{A_{f}}}\left|\beta\left(\underline{h}\left(m g_{f}^{-1}\right)\right)\right|_{A}^{1 / 2} \xi_{j}\left(g_{f}\right) d g_{f} d m .
\end{aligned}
$$

Similarly we obtain the following inequality for $s \in J$ :

$$
\begin{aligned}
& \left\|s \cdot\left(F * \xi_{j}\right)^{\wedge}\left(s, \chi^{-1}, \tau\right)\right\|_{\tau} \\
& \quad \leqq C_{2} \operatorname{dim} \tau \int_{M} \int_{G_{A_{f}}}\left|\beta\left(\underline{h}\left(m g_{f}^{-1}\right)\right)\right|_{A}^{1 / 2} \xi_{j}\left(g_{f}\right) d g_{f} d m .
\end{aligned}
$$

On the other hand, it is known that, for any $s \in J$,

$$
\|\Phi(s, \chi, \tau)\|_{\tau}=C_{3} \text {. }
$$

Here $C_{3}$ is a positive constant which depends only on $\tau$ and $\chi$ (cf. [5], $\S 6,(6.16)$ ). Combining (3.21), (3.24), (3.25) and (3.26), we obtain the inequality (3.19) if we set $C=\left(C_{1}+C_{2} C_{3}\right) \operatorname{dim} \tau$. Hence the lemma has been proved.

We set, for $s \in C$,

$$
\Omega\left(s, \xi_{j}\right)=\int_{M} \int_{G_{A_{f}}}\left|\beta\left(\underline{h}\left(m g_{f}^{-1}\right)\right)\right|_{A}^{s} \xi_{j}\left(g_{f}\right) d g_{f} d m .
$$

By virtue of Lemma 8, the proof of (3.18), and hence of Proposi- 
tion 4, has now been reduced sto the verification of the following proposition.

Proposition 6. If (0.1) is satisfied, then we have

$$
\lim _{j \rightarrow \infty} \Omega\left(\frac{1}{2}, \xi_{j}\right) / \operatorname{deg} \xi_{j}=0 \text {. }
$$

Proof. To prove the proposition, we shall express $\Omega\left(s, \xi_{j}\right)$ as a product of some integrals of zonal spherical functions on $G_{k_{8}}=$ $\mathrm{SL}\left(2, k_{\mathfrak{g}}\right)$ for $\mathfrak{g} \in \mathscr{S}$. For $\mathfrak{g} \in \mathscr{P}_{f}$, we fix the Iwasawa decomposition of $g_{\mathrm{g}} \in G_{k_{\mathrm{g}}}$ given by $g_{\mathrm{g}}=\underline{u}\left(g_{\mathrm{g}}\right) \underline{h}\left(g_{\mathrm{g}}\right) \underline{m}\left(g_{\mathrm{g}}\right)$, where $\underline{u}\left(g_{\mathrm{g}}\right) \in U_{k_{\mathrm{g}}}, \underline{h}\left(g_{\mathrm{g}}\right) \in H_{k_{\mathrm{g}}}$, and $\underline{m}\left(g_{8}\right) \in M_{8}$. We set

$$
\beta(h)=t^{2} \text { for } h=\left(\begin{array}{c}
t \\
t^{-1}
\end{array}\right) \in H_{k_{8}} .
$$

We denote by $\mid l_{8}$ the module of $k_{8}$. Namely, for a prime element $\kappa$ of $k_{8}, n \in \boldsymbol{Z}$, and for any element $\varepsilon$ in the unit group of $O_{8}$, we put

$$
\left|\kappa^{n} \varepsilon\right|_{8}=q^{-n} \text {. }
$$

Here $q$ denotes the order of the residue field of $k_{9}$. We normalize the Haar measure $d m_{\mathfrak{s}}$ on $M_{\mathfrak{g}}$ so that

$$
\int_{M_{\mathrm{g}}} d m_{\mathrm{g}}=1
$$

We set, for $g \in G_{k_{\mathrm{g}}}$ and $s \in \boldsymbol{C}$,

$$
\omega_{\mathrm{g}}(g, s)=\int_{M_{\mathrm{g}}}\left|\beta\left(\underline{h}\left(m_{\mathrm{g}} g\right)\right)\right|_{\mathrm{g}}^{s} d m_{\mathrm{g}} .
$$

The integral (3.27) converges absolutely for any $s \in C$, and for any $g \in G_{k_{8}}$. We call $\omega_{s}(g, s)$ the zonal spherical function on $G_{k_{8}}$. This function is, as a function of $g, M_{g}$-biinvairant on $G_{k_{8}}$.

For $m=\Pi_{v} m_{v} \in M$ and $g_{f}=\Pi_{b \in \mathscr{S}_{f}} g_{\mathrm{g}} \in G_{A_{f}}$, it is easily verified that

$$
\left|\beta\left(\underline{h}\left(m g_{f}^{-1}\right)\right)\right|_{A}=\prod_{g} \in \mathscr{G}_{f}\left|\beta\left(\underline{h}\left(m_{g} g_{g}^{-1}\right)\right)\right|_{\mathfrak{g}} .
$$

It follows that

$$
\begin{aligned}
& \Omega\left(s, \xi_{j}\right)=\prod_{\mathfrak{g} \in \mathscr{S}} \int_{M_{\mathfrak{g}}} \int_{S_{\mathfrak{g}}(j)}\left|\beta\left(\underline{h}\left(m_{\mathfrak{g}} g_{\mathfrak{g}}^{-1}\right)\right)\right|_{\mathfrak{g}}^{s} d g_{\mathfrak{g}} d m_{\mathfrak{g}} \\
& \times \prod_{\mathfrak{g} \in \mathscr{S}_{f} f^{-S}} \int_{M_{\mathfrak{g}}} \int_{M_{\mathfrak{g}}}\left|\beta\left(\underline{h}\left(m_{\mathrm{g}} g_{\mathfrak{g}}^{-1}\right)\right)\right|_{\mathbb{g}}^{8} d g_{\mathrm{g}} d m_{\mathfrak{B}} .
\end{aligned}
$$

Note that $g_{8} \in M_{8}$ implies $\left|\beta\left(\underline{h}\left(m_{8} g_{8}^{-1}\right)\right)\right|_{8}=1$ for any $m_{8} \in M_{8}$. Thus 


$$
\Omega\left(s, \xi_{j}\right)=\prod_{\mathfrak{g} \in \mathscr{S}} \int_{\mathbb{M}_{\mathbb{8}}} \int_{S_{\mathfrak{g}}(j)}\left|\beta\left(\underline{h}\left(m_{\mathfrak{g}} g_{\mathfrak{B}}^{-1}\right)\right)\right|_{8}^{s} d g_{8} d m_{\mathfrak{8}} .
$$

Changing the order of integrations, we obtain

$$
\Omega\left(s, \xi_{j}\right)=\prod_{\mathfrak{g} \in \mathscr{P}} \int_{S_{\mathfrak{g}}(j)} \omega_{8}\left(g_{\mathfrak{g}}^{-1}, s\right) d g_{\mathfrak{g}} .
$$

Applying Lemma 5, we observe that it is enough to establish the following.

Lemma 9. For every $\mathfrak{g} \in \mathscr{P}_{f}$, the function on $G_{k_{8}}$ given by $g \mapsto$ $\omega_{8}(g, 1 / 2)$ vanishes at infinity.

Proof. As is well-known, the zonal spherical function $\omega_{8}(g, 1 / 2)$ is a matrix coefficient of an irreducible unitary representation of $\mathrm{SL}_{2}\left(k_{8}\right)$ belonging to the principal series. Hence the lemma follows from the general result of Howe and Moore (stated in $\S 2$ as Lemma 4). However, in the following, we give a direct proof of the lemma based on the precise knowledge on the behavior of $\omega_{8}(g, 1 / 2)$ on $G_{k_{8}}$.

By virtue of the explicit formula for the zonal spherical function on $G_{k_{g}}$ (cf. [4], Chap. II, $\S 3.10$ ), we have

$$
\omega_{8}\left(\left(\begin{array}{cc}
\kappa^{n} & \\
\kappa^{-n}
\end{array}\right), \frac{1}{2}\right)=q^{-n}(1+q)^{-1}\{(2 n+1) q-(2 n-1)\}
$$

for $n \geqq 0$. Hence

$$
\lim _{n \rightarrow \infty} \omega_{8}\left(\left(\begin{array}{cc}
\kappa^{n} & \\
& \kappa^{-n}
\end{array}\right), \frac{1}{2}\right)=0 .
$$

Then the lemma follows from (3.28) together with the Cartan decomposition of $G_{k_{\mathrm{g}}}$ :

$$
G_{k_{8}}=\bigcup_{n=0}^{\infty} M_{8}\left(\begin{array}{c}
\kappa^{n} \\
\kappa^{-n}
\end{array}\right) M_{8} \text { (disjoint union) . }
$$

Thus Theorem 2 has been completely proved.

\section{REFERENCES}

1. I. N. Bernshtein, All reductive p-adic groups are tame, Functional Analysis and its Appl., 8 No. 2, (1974), 91-93.

2. A. Borel, Some finiteness properties of adele groups over number fields, Publ. Inst. Hautes Etudes Scient., 16 (1963), 5-30.

3. A. Borel and J. Tits, Homomorphismes "abstraits" de groupes algébriques simples, Ann. of Math., 97 (1973), 499-571.

4. I. Gel'fand, I. Graev and I. I. Pjateckii-Šapiro, Representation Theory and Automorphic Functions, Saunders Company. 
5. R. Godement, Analyse spectrale des fonctions modulaires, Séminaire Bourbaki, Exposé 278, 1964.

6. H. Hijikata, On the structure of semi-simple algebraic groups over valuation fields, I, Japan. J. Math., 1, No. 2, (1975), 223-300.

7. R. Howe and C. C. Moore, Asymptotic properties of unitary representations, J. Functional Analysis, 32 (1979), 72-96.

8. J. E. Humphreys, Linear Algebraic Groups, Springer-Verlag.

9. M. Kneser, Strong approximation, Algebraic groups and discontinuous subgroups, (Proc. Symp. Pure Math. Boulder, Colo.), (1965), 187-196.

10. M. Kuga, On a uniformity of distribution of 0-cycles and the eigenvalues of Hecke's operators, I, II, Coll. Gen. Ed. Sci. Papers, Univ. Tokyo, 10 (1960), 1-16, 171-186.

11. V. P. Platonov, The problem of strong approximation and the Kneser-Tits conjecture for algebraic groups, Math. USSR-Izvestija, 3 (1969), No. 6, 1139-1147.

12. - Addendum to the paper "The problem of strong approximation and the Kneser-Tits conjecture for algebraic groups", Math. USSR-Izvestija, 4 (1970), No. 4, 784-786.

13. C. Pommerenke, Uber die Gleichverteilung von Gitterpunkten auf m-dimensionalen Ellipsoiden, Acta Arith., 5 (1959), 227-257.

14. G. Shimura, Arithmetic of alternating forms and quaternion hermitian forms, J. Math. Soc. Japan, 15 (1963), 33-65.

15. A. Weil, Basic Number Theory, Springer-Verlag.

16. H. Yoshida, On an analogue of the Sato Conjecture, Inventiones Math., 19 (1973), 261-271.

Received July 9, 1979.

UNIVERSITY OF TOKYO

BUNKYOKU, TOKYo, JAPAN 



\section{PACIFIC JOURNAL OF MATHEMATICS}

\section{EDITORS}

DONALD BABBITT (Managing Editor)

University of Galifornia

Los Angeles, California 90024

Hugo Rossi

University of Utah

Salt Lake City, UT 84112

C. C. MOORE AND ANDREW OGG

University of California

Berkeley, CA 94720
J. DugundjI

Department of Mathematics University of Southern California Los Angeles, California 90007

R. Finn and J. Milgram Stanford University Stanford, California 94305

ASSOCIATE EDITORS

E. F. BECKENBACH

B. H. NeumanN

F. WOLF

K. YoSHIDA

\section{SUPPORTING INSTITUTIONS}

UNIVERSITY OF BRITISH COLUMBIA

UNIVERSITY OF SOUTHERN CALIFONIA

CALIFORNIA INSTITUTE OF TECHNOLOGY

UNIVERSITY OF CALIFORNIA

MONTANA STATE UNIVERSITY

STANFORD UNIVERSITY

UNIVERSITY OF HAWAII

UNIVERSITY OF NEVADA, RENO

UNIVERSITY OF TOKYO

NEW MEXICO STATE UNIVERSITY

UNIVERSITY OF UTAH

OREGON STATE UNIVERSITY

WASHINGTON STATE UNIVERSITY

UNIVERSITY OF OREGON

UNIVERSITY OF WASHINGTON 


\section{Pacific Journal of Mathematics \\ Vol. 88, No. $1 \quad$ March, 1980}

Michael James Beeson, Extensionality and choice in constructive mathematics .................................... 1

José L. Blasco Olcina, Two questions on Wallman rings.............. 29

Peter I. Booth and J. Tillotson, Monoidal closed, Cartesian closed and convenient categories of topological spaces .................. 35

Peter B. Borwein, Rational functions with positive coefficients, polynomials and uniform approximations ......................... 55

Josip Globevnik, Fourier coefficients of the Rudin-Carleson extensions . . . . 69

Jacob Kofner, Quasimetrizable spaces ...................... 81

Mark Mandelker, Resolutions on the line ..................... 91

Lynn McLinden, An analogue of Moreau's proximation theorem, with application to the nonlinear complementarity problem ............ 101

Atsushi Murase, On the uniform distribution property of certain linear algebraic groups ................................ 163

Nicholas Th. Varopoulos, Zeros of $H^{p}$ functions in several complex

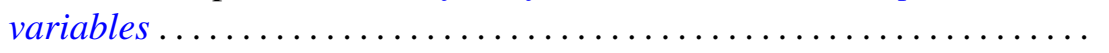

